\title{
LONG-FOCUS CCD ASTROMETRY OF PLANETARY
}

\section{SATELLITES}

\author{
D.PASCU \\ U.S.Naval Observatory
}

\begin{abstract}
.
CCD detectors are rapidly replacing the photographic plate and photomultiplier in satellite observations used for orbital improvement. This includes both phenomena timings as well as tangent plane astrometry. In most cases this change has been for the better, but in some areas there has been no gain - even a loss. We will review this change in terms of the recent history of satellite observations. The impact of the CCD will be discussed in terms of its applications, and the increase in precision it affords. Finally, a few things will be said about future directions, especially about spin-off applications.
\end{abstract}

\section{RECENT HISTORY}

The modern period of astrometric satellite observing began about 1965, anticipating the spacecraft reconnaissance of the outer planets, but apparently not motivated by it. Rather, it resulted directly from the need to improve the badly out-of-date ephemerides published in the national almanacs. Photographic observing programs were begun, principally at the Naval Observatory in the United States, and at the Pulkova Observatory in the Soviet Union, with an emphasis on the Martian satellites (because of an interest in the secular acceleration of Phobos) and the Galilean moons of Jupiter.

It became clear to the planners of NASA's Grand Tour of the outer planetary systems that the accuracy of the existing satellite ephemerides was incompatible with the more severe requirements of space reconnaissance. The observational effort was greatly expanded, driven largely by 
NASA funding (Seidelmann 1977, 1979). This period of expansion lasted throughout the Voyager years, 1973 - 1989. While photographic techniques predominated (Pascu 1977, 1979), this period saw the introduction of several new observational methods - in particular, mutual phenomena techniques (Millis 1974; Aksnes and Franklin 1975), space techniques (Born and Duxbury 1975; Duxbury and Callahan 1988, 1989), and CCD techniques (Pascu et al. 1983, 1987).

At present, space reconnaissance is still the major driver of satellite observation, but there is also a broader component to this demand. Much of it is fueled by an interest in the physical study of the satellites using the Hubble Space Telescope (HST), and more accurate ephemerides are required for such observations. An added complication is that many of these satellites were discovered by the Voyager spacecrafts and cannot be observed from the ground. Consequently, the astrometry of these satellites must also be done with HST. CCD techniques are now in general use, but a number of promising techniques have appeared in the past few years. HST has already been applied to the astrometry of the faint satellites of Pluto (Null et al. 1993), Uranus (Zellner et al. 1994; Currie et al. 1994; Pascu et al. 1995), and Saturn (Bosh and Rivkin 1995; Showalter et al. 1995). Infrared techniques have proved useful for ground-based observations of the inner systems of Jupiter and Saturn (Nicholson and Matthews 1991; Nicholson et al. 1992), and may have some limited application to the brightest of the inner satellites of Uranus and Neptune. The radar technique (Campbell et al. 1978) and the Very Large Array technique (Muhleman et al. 1986) are beyond the scope of this review, but are mentioned because they produce highly accurate positions.

\section{OBSERVATION TYPES}

CCDs are applied to two observation types - astrometric and phenomena. Astrometric observation refers to the measurement of relative coordinates, rectangular or polar, in the tangent plane. The measurements can be intersatellite (one satellite referred to another) or with respect to the planet. The simple observations themselves have generally been reported, rather than compound observations such as normal points. This is the most common observation type since it can be applied to all satellites, at all apparitions. It has the additional advantage that a full complement of orbital parameters can be determined, in particular, the semi- major axis from which the system mass is obtained. A recent example of this is the Pluto-Charon mass (Tholen and Buie 1995). The major drawback to this observation type is its need for large instruments, both in aperture and focal length.

Phenomena observation refers to the measurement of the epoch of an 
event, such as an eclipse or occultation of a satellite. This event can be due to the planet or it can be due to another satellite (a mutual event). The event can be measured photometrically or geometrically (astrometrically) in the case of a mutual occultation. The "epoch" refers to some identifiable part of the event, such as the instant of minimum light, or central eclipse for a photometric event, or the instant of minimum separation in the case of an astrometrically measured mutual occultation. This epoch is sometimes referred to as the "mid-event time." Photometric phenomena observations have the advantage that they can be made with relatively small telescopes (Mallama 1992a,b) and are independent of instrumental parameters. It is for these reasons that relatively precise phenomena observations of the Galilean satellites, due to Jupiter, have been made for more than three centuries. Another notable success of the phenomena technique was the determination of the orbital motions in the Pluto- Charon system from observations of their mutual phenomena during the late 1980s. For the Galilean moons however, phenomena observations due to Jupiter are not of the positional accuracy of the mutual phenomena or the astrometric observations (Lieske 1995b). The principal drawback of the mutual phenomena technique is the rarity of events for the great majority of satellites. In addition, there is a limitation on the precision attainable for the orbital orientation parameters, and the orbital scale - the semi-major axis - can not be determined at all. While the mutual phenomena technique provided the means for analyzing the motions in the Pluto-Charon system, it was the astrometric technique, applied with the HST, which produced an accurate scale for the system, and thus an accurate system mass (Tholen and Buie 1995).

\section{IMPACT OF CCDs}

The CCD has had a favorable impact on both astrometric and phenomena observations. The high quantum efficiency ( 70 percent) benefitted the astrometric technique the most. Now, even twentieth magnitude satellites can be imaged without differential guiding, and a higher signal-to-noise ratio means higher astrometric precision for the faint moons. For the mutual phenomena technique, also, the high quantum efficiency means that the technique can be applied to fainter satellites, as in the Saturnian system.

Linear response and area photometry are the two features which make the CCD especially applicable to the observation of the photometric mutual phenomena; both phenomena satellites and reference objects can be recorded simultaneously. And, for both phenomena observation and astrometry, one can account for the scattered light from the primary more precisely. 
But, the CCD also has several limitations: The small size of the CCD chip makes it difficult to calibrate scale, and especially the orientation. Even in small standard fields (cluster fields), relative star positions cannot be measured with enough precision to compete with a field which is linearly an order of magnitude greater (on a photographic plate). The reason that orientation is more difficult to calibrate, is that the orientation of the chip must be determined at the point of observation, or else the polar axis alignment must be calibrated.

The small size of the chip also makes it unlikely that enough reference stars will appear on the frame to provide spherical equatorial coordinates (R.A., Dec.) for objects in the field. For some applications, the size problem can be solved with a mosaic of CCDs, but other problems, which require a single chip, must await the arrival of the large 8-cm chips which are expected in a few years (Harris 1995).

Since the CCD chip is not only the detector but the measuring machine as well, the astrometric integrity of the chip is a major consideration. The astrometric integrity of the chip has at least four components, three structural and the fourth electronic. Its flatness, the orthogonality of its rows and columns, and the thickness and spacing of the rows and columns are the structural components. Many of the early chips were crinkled or bowed, while others were rhomboidal (Harris 1995). The electronic component has to do with image form factors. If the image is distorted by blocked columns, or charge transfer inefficiency, for example, the centroid will not represent its position well. Because of charge transfer inefficiency, the Mark IV TI 800 $\mathrm{X} 800 \mathrm{CCD}$, prototype of the HST CCDs (now in use at the USNO), is used well below full well for the highest astrometric precision (Dahn 1994). If a CCD is to be used for astrometry, it should be constructed and calibrated in the same manner as were the metrics of the modern plate measuring machines. While most of these problems can be solved, they need to be addressed in the specifications and construction of the CCD.

The photometric integrity of the CCD is also a consideration. While it also has several components, the most important for photometric observations is the linearity. Few CCDs are linear over their entire dynamic range and need to be calibrated.

Brightness attenuation and magnitude compensation are problematic with the CCD. The inaccessibility of the focal plane (chip) makes it difficult to reduce the brightness of the planet without reimaging. An alternative is a prefocal stop, which limits the distance from the planet at which a satellite can be observed. Another is placing the attenuator inside the dewar, either directly on the CCD chip or on a pellicle placed immediately before the chip. 


\section{APPLICATIONS AND TECHNIQUES}

\subsection{PHENOMENA}

Observations of phenomena due to the planet refer to eclipses of the Galilean satellites by Jupiter (the eclipse/occultation events of Pluto and Charon are more properly classified as mutual events). As mentioned above, positional information derived from the Jupiter events are no longer competitive in accuracy with that from mutual phenomena or astrometric observations. This is not a calibrational problem, but due to an inability to suitably model the event. Although Mallama $(1991,1992 b)$ is making some progress in this area, he suggests that the future value of such observations appears to be in deriving information about the atmosphere of Jupiter itself (Mallama 1991, 1995).

Observations of the photometric mutual phenomena, however, are an entirely different matter. Since their first modern observation in 1973 it was recognized that this observation type would yield highly accurate positional data (Brinkmann and Millis 1973). The mutual events of the Galileans have been observed photometrically every six years, beginning in 1973. Since 1979 the Bureau des Longitudes has organized these observations as international campaigns involving many observatories (Arlot et al. 1982; Arlot and Thuillot 1988; Arlot et al. 1990). The next series of events will occur in 1996, and plans are already underway for a campaign to observe those (Arlot 1995). With the widespread use of CCDs at those events, it will be possible to measure the events astrometrically as well as photometrically. Astrometric phenomena were first mentioned by Arlot (1982) and attempted by Arlot et al. (1982) at the 1979 mutual events with promising results.

Likewise, the serendipitous discovery of Charon by Christy (1978), and the fortuitous recognition by Andersson (1978) that mutual phenomena were imminent, led to six years of observations of Pluto- Charon (photometric) events which were invaluable to the determination of physical parameters of Pluto and Charon, and to the study of the motions in that system. The Pluto-Charon events ended in 1990 and will not occur again for more than a century. Buratti et al. (1995) describe the CCD observations of these events made with the Palomar 60-inch telescope, and give references for most of the others.

During 1995, activity will be focussed on the mutual events of the Saturnian satellites which occur every 15 years. These were last observed at the ring-plane crossing in 1980 (Dourneau 1982; Aksnes et al. 1984). The Bureau des Longitudes organized an international campaign to observe these events in 1995 (Arlot and Thuillot 1993). These phenomena are more difficult to observe than the Galilean events, but the introduction of CCD 
detectors should ensure their success. While CCDs were used for the PlutoCharon events as early as 1985 (see Buratti et al. 1995), they were not applied to the Galilean satellites until the 1990-91 mutual events (Colas 1991; Mallama 1992a; Le Campion et al. 1992).

Calibration of the CCD, for photometric use, involves bias subtraction (zero point of pixel counters), flatfielding (correction for variation in pixel sensitivity) and determination of linearity (finding the range of digital counts in the pixels in which these counts are linearly proportional to the incident light). Dark current is not significant for cooled CCDs and short exposures, but for the infrared devices, dark current must also be taken into account. Bias subtraction is fairly straightforward, but flatfielding is not, and its effects on phenomena observations has not been investigated. For the bright Galilean satellites events, this is probably not a problem, but it may be for the total mutual eclipses of the Saturnian events. Because of the large dynamic range of the total eclipses in the Saturnian system, calibration of the linearity of the CCD will also be important. For small dynamic range events, this is not a major issue.

\subsection{ASTROMETRY}

Astrometric CCD observation of the planetary satellites is widespread, with major programs in the United States (Pascu et al. 1983, 1987, 1992b, 1995; Pascu 1994b; Monet and Monet 1992, A. Monet 1993; Nicholson and Matthews 1991; Nicholson et al. 1992; Rohde and Pascu 1993, 1994), France (Colas and Arlot 1991; Colas 1992, 1994, 1995; Arlot et al. 1989, 1994; Rapaport 1994; Viateau and Rapaport 1995; Le Floch 1994), and Great Britain (Beurle et al. 1993; Harper 1994, 1995; Jones 1995), and smaller programs in Brazil (Vieira Martins and Veiga 1995), Spain (Lopez-Garcia 1994), and Russia (Zamarashkin et al. 1994). Since CCD astrometry is now applied to all of the satellites, the observational techniques of detection and calibration are more varied than for the phenomena.

The most difficult problem of detection is that of a faint satellite near a bright primary. A Lyot-type coronagraph appears to be the best solution to the problem (Baum et al. 1981; Pascu et al. 1983) because it eliminates the diffracted light and makes it possible to attenuate the light of the primary as well. While apodizing diaphragms can be designed to remove the diffraction spikes caused by the secondary supports, the diffraction caused by the entrance pupil is often increased. If prefocal stops are used to attenuate the light of the primary, they must be placed very close to the CCD chip or they will limit the distance from the planet that a satellite can be observed astrometrically (Vieira Martins and Veiga 1995). The most heroic detection was that of Proteus by Colas and Buil (1992). The technique is 
promising but needs much work to make it astrometrically viable. Special techniques are also needed for satellites which are too bright. Astrometric CCD observations of the Galilean satellites made at the U.S. Naval Observatory use dark filters to lengthen the exposure times to a few seconds to help average the effects of seeing. Additional averaging is accomplished by combining numerous frames into normal points (Monet and Monet 1992; A. Monet 1993; Owen 1995).

Assuming that one has an astrometric grade CCD, or one that is adequately mapped and calibrated, the three remaining calibrational problems are scale, orientation and coordinate origin determination (Pascu 1977). Since CCDs are small and star catalogs have a too low density, plate constants are unlikely until the large chips and denser catalogs become available. This means that some version of the trail/scale method must be used - although the diminutive size of the CCD is less than ideal for accurate results. The focal plane scale has been determined in several ways. The best general way is to use a small scale field in a cluster. Astrometric sequences in M15, M92 and Pleiades are most commonly used. The problem is that astrometric standard fields are not available for all parts of the sky. Veiga and Vieira Martins (1994) used the motion of Uranus to determine both scale and orientation. If one is interested primarily in faint, inner satellites, for which the CCD is best adapted, then one may use the brighter satellites of the system to calibrate the CCD frames. The best example of this is the technique of Veillet and Ratier (1980) who used the ephemeris positions of the four bright moons of Uranus to implement a plate constants solution for scale, orientation and coordinate origin to obtain positions for Miranda. The same method was applied successfully with a CCD by Pascu et al. (1987). While the configuration around Uranus is unique, both the Jovian and Saturnian systems have at least two well-spaced bright satellites whose relative ephemeris positions are accurate to $\mathbf{3 0}$ mas or better. Such configurations will give scale, orientation and coordinate origin. The use of wide double star pairs is not suitable for accurate scale or orientation calibrations.

Calibration of CCD orientation is more problematic, as explained in Section 3. The most general method for calibration is to record star trails at the same declination as the planet. While it is understood that the short lengths of the trails will fail to produce the accuracy of the longer trails on photographic plates, this disadvantage is reduced by the sensitivity of the CCD, which permits one to record many trails by simply stopping the drive. Some observers have used the scale field clusters to calibrate both scale and orientation. This will work only if the cluster and planet are at the same declination. Otherwise, a systematic error will result due to polar axis misalignment. Jones (1995) has attempted to calibrate this misalignment 
so that orientation may be calibrated in scale fields as well. As for scale calibration, the brighter moons can also be used to calibrate orientation, and coordinate origin if only the fainter moons are required.

Coordinate origin (reference object) determination is in most cases simple, but in a few instances it is quite involved. For the great majority of the satellites, only intersatellite observations are feasible. And for most satellites, there is at least one other satellite of comparable brightness, or at least within the spatial or dynamic range of the CCD (about 6 magnitudes), to provide a reference zero-point. The exceptions are: the outer moons of Jupiter and Saturn, the Tethys and Dione Lagrange librators, Triton and Nereid. The solution for the outer satellites is to image them only when three or more HST Guide Star Catalog (GSC) stars are in the field (about ten arcmin across). The R.A. and Dec. obtained in this way can be compared to the ephemeris position of the planet. While the GSC star positions are not precise enough to calibrate the scale and orientation, the positions derived in this way are as good or better than the photographic because the images are not trailed in the shorter CCD exposures. For the remainder of the problem satellites, a neutral density filter can solve the problem if a coronagraph is used in the observations. With or without a coronagraph, the method suggested by Pascu et al. (1983) can be used successfully. In that method, shorter exposures of the brighter satellites are taken alternately with the longer exposures of the faint satellites. The motions on the CCD frames (in pixel units) of the bright satellites, as a function of time, are determined from these short exposures. The positions of these bright moons can then be interpolated from these functions for the mean times of the long exposures. This gives, finally, the positions of the faint satellites relative to the bright ones on the long exposure frames.

\section{ACCURACY}

There is no issue in satellite astrometry more contentious than the claims of observational accuracy among the various techniques. Much of this is due to the mistaken comparison of dissimilar quantities, such as the error of a single observation with that of a normal point or other compound quantity. Another invalid comparison is the internal precision of one method with the external precision of another. A third problem is the failure to take into consideration other technique-independent sources of astrometric error, such as the increase in error with the angular separation of the measured bodies (Pascu et al. 1991; Pascu 1994a). Only the external error (precision) of one observation (i.e. the rms residual from a definitive orbit) should be used in these comparisons, and proper consideration should be given to independent parameters, such as separation of the two satellites and 
the integration time. Comparisons of this type would not only indicate directions to improvement, but would show that most modern observational techniques are competitive.

The mutual phenomenon "mid-event time," the quantity used in the orbital adjustment, is not, strictly speaking an observation. It is a quantity derived from numerous photometric or astrometric observations by a leastsquares procedure which could rival that of the orbital adjustment itself. It has been referred to here as a compound observation akin to a normal point, although technically, only one observation is necessary if only the mid-event time is determined. In that case, the mid-event time would be comparable to a single observation. Recent estimates of the accuracy of the photometric mutual phenomena (compound observations) of the Galilean moons were made by Morando and Descamps (1994), and by Lieske (1995b). Morando and Descamps reported preliminary errors of 28 mas $(100 \mathrm{~km})$ for data uncorrected for the deviation of center of light from center of figure, and 4 mas $(15 \mathrm{~km})$ for corrected data. Lieske reports a rms residual of 30 mas $(100 \mathrm{~km})$ for the corrected data with "wide differences for the same event by different observers." These results suggest systematic errors in the observations and/or non- standard procedures for reduction of these complex data; and there is good evidence for the latter (Lieske 1995a). In any case, it appears that with the introduction of CCD techniques and improved reduction procedures, the best observations of the photometric mutual events of the Galilean satellites will produce data with an external precision in the range of 10 to 30 mas.

Sources of systematic error in the astrometric observations are due to uncalibrated scale and orientation errors and to a non-astrometric CCD chip. In the first case, the residuals increase with separation of the satellites measured. While these can be significant for CCD observations because of the difficulty in calibrating a diminutive focal plane, relative observations of satellites of small angular separation will be negligibly affected (Pascu et al. 1991; Pascu 1994a). A non-astrometric CCD chip is more problematic and should be mapped or avoided.

The external accidental rms error ranges from 20 to 120 mas for a single ground-based observation. This value usually includes the systematic error, if any. It also varies with separation of the measured satellites, and with the integration time and signal-to-noise ratio as well (as affecting the centroiding precision). Centroiding precision is about 5 - 20 mas, leaving the remainder to some limiting physical factor related to the separation and integration time. This limiting factor is believed to be image motion caused by atmospheric seeing. Lindegren (1980) relates the mean error, m.e. (arcsec), in the measured separation, S (radians), of two images, and integration time, $\mathrm{T}(\mathrm{sec})$ by 


$$
\text { m.e. }=1.3 S^{0.25} T^{-0.5}
$$

This formulation is a somewhat optimistic representation of the mean errors of CCD as well as photographic observations. It also demonstrates why any gain in precision due to the CCD would be primarily for the fainter satellites; the CCD is so sensitive that the bright satellite images saturate before the seeing excursions have time to average out. One must resort to attenuation and normal points, as described earlier.

For Hubble Space Telescope observations, the accuracy is limited by the measuring precision. Separation measurements of images near full well are expected to be accurate to 2 mas in the Planetary Camera. The accuracy for faint images is about 5 - 20 mas (Pascu et al. 1995). However, in the final analysis, for both the phenomena and astrometric observations, the astrometric precision of the observation depends on how accurately one can correct for the difference between the center of light, which is measured, and the center of figure (assumed to be at the center of gravity). Descamps (1992) and Mallama (1991) have had considerable success in developing algorithms for doing just that. They used Voyager images, observed photometric models and light scattering theory to compute corrections to centerof-light observations of the Galilean satellites. The ultimate precision for observations of the Galilean moons can be estimated from the rms residual given by Lieske (1995b) for the Voyager observations which were rigorously center-of-figure. He reports $45 \mathrm{~km}$ (15 mas). This suggests that the greatest astrometric precision possible will be for the smallest satellites!

\section{NEAR FUTURE}

CCD use in satellite observation can only increase in the near future. In the next few years, much activity will center about the mutual events of the moons of Saturn and the Galilean moons of Jupiter. There will also be considerable activity with HST observations of the faintest satellites not visible from the ground. To date only the inner satellite system of Neptune has not been imaged with HST and that is expected to change in two years.

In about five years, $9 \mathrm{k} \times 9 \mathrm{k}$ CCD chips are expected to be available. It will then be possible to make CCD observations of the larger satellite systems with an accuracy comparable to the photographic ones, because scale calibration can then be done with standard scale fields, and orientation determined from longer trails. In fact, when the Tycho or faint star catalogs become available, it will be possible to implement a plate constants solution - calibrating both parameters simultaneously.

The ring-skimming satellites of Jupiter - Metis and Adrastea - are best observed from the ground at 2-microns (Nicholson and Matthews 1991), 
and periodic, if not regular, observations should be made. The IR technique should also be developed and extended to Puck and Proteus.

Some of the more interesting possibilities for future CCD observations lie in spin-off applications. When it is possible to implement a plate constant solution using the 9k x 9k CCDs and Tycho stars, it will then be feasible to obtain a five-fold increase in the positional accuracy of the planets (R.A. and Dec.) from relative observations of their satellites (Pascu and Schmidt 1990). These would be on the Hipparcos Catalog system and have an estimated precision of 30 mas. While this could be done better photographically for Jupiter (because of attenuation problems) and possibly for Saturn, certainly the advantage would go to the CCD for Uranus, Neptune and Pluto.

Another spin-off of the differential astrometry is differential photometry. The same observational strategy and data, used to obtain the astrometric results, can be used to obtain magnitudes, colors, albedos and light curves of the faint satellites from accurately known photometric parameters of the bright satellites (Pascu et al. 1992a, 1993, 1994a, 1995; Pascu and Rohde 1993). These data in turn will reveal information on the rotation of the satellites and their surface composition, as well as information on the severe conditions and processes in the strong magnetospheres of their primaries.

An ingenious spin-off of the mutual event observations was the monitoring and mapping of the volcanos and hot spots on Io from infrared photometry of the mutual occultations of Io. Goguen et al. (1988) applied this technique first at the 1985 mutual events using a photometer. Descamps et al. (1992) improved the technique at the 1991 events by using a CCD and combining simultaneous infrared and blue observations. The blue observations were used to derive corrections to the ephemerides of Io and the occulting moon, resulting in a more precise mapping of the volcanos.

\section{SUMMARY AND CONCLUSIONS}

The demand for high quality observations of the planetary satellites remains high, motivated by the needs of space reconnaissance and other astrometric projects. The principal advance in astrometric observation in the past 15 years was the introduction of CCD detectors. The quantum efficiency and linear response of the CCD detector have been largely responsible for its success, both for tangent plane astrometry and for the observation of the mutual phenomena of the bright moons of Jupiter and Saturn. At present, the CCD has been applied to all satellites detectable from the ground or with HST, except the inner system of Neptune, and there are substantial observing efforts in several countries. However, caution should be used when applying CCDs to large scale systems because the small size and complexity 
of the CCD chip make it difficult to calibrate. In its present configuration, the CCD is best suited to the observation of faint satellites near their primaries. This caveat notwithstanding, it is now possible to obtain relative positions of the satellites with a precision in the range 2 to 30 mas for HST observations, while a precision of 10 to 30 mas can be reached for groundbased observations of mutual phenomena or astrometric observations made when two satellites are at small separation $(<50$ arcsec). The limitation on precision appears to depend on how well the positions of the centers of light are known relative to their centers of figure. CCDs also offer several spin-off applications to the satellite astrometry. The volcanos of Io have been monitored and mapped with observations of the photometric mutual phenomena, while color photometry of the faint satellites relative to the bright ones result from the astrometric measurements.

\section{References}

Aksnes, K., and Franklin, F.A. (1975). "Mutual Phenomena of the Galilean satellites in 1973. I. Total and near-total occultations of Europa by Io," Astron. J. 80, 56.

Aksnes, K., Franklin, F., Millis, R., Birch, P., Blanco, C., Catalano, S., and Pirronen, J. (1984). "Mutual phenomena of the Galilean and Saturnian satellites in 1973 and 1979/80," Astron. J. 89, 280.

Andersson, L.E. (1978). "Eclipse phenomena of Pluto and its satellite," Bull. Amer. Astron. Soc. 10, 586.

Arlot, J.-E. (1982). "Amélioration des Éphémérides des Satellites Galiléens de Jupiter par l'Analyse des Observations," Thèse de Doctorat d'Etat, Bureau des Longitudes.

Arlot, J.-E. (1995). personal comm.

Arlot, J.-E., Bernard, A., Bouchet, P., Daguillon, J., Dourneau, G., Figer, A., Helmer, G., Lecacheux, J., Merlin, Ph., Meyer, C., Mianes, P., Morando, B., Naves, D., Rousseau, J., Soulie, G., Terzan, A., Thuillot, W., Vapillon, L., and Wlerick, G. (1982). "Les resultats de la campagne d'observation PHEMU79 des phenomenes mutuels des satellites galileens de Jupiter en 1979," Astron. Astrophys. 111, 151.

Arlot, J.-E., Bouchet, P., Gouiffes, Ch., Schmider, F.X., Thuillot, W. (1989). "Mutual Events of the Galilean Satellites: An Analysis of the Observations Made in 1985 at ESO," Astron. J. 98, 1890.

Arlot, J.-E., Colas, F., Thuillot, W. and Vu, D.T. (1994). "CCD observations at the Bureau des Longitudes: analysis of the positions of satellites," in Galactic and Solar System Optical Astrometry (L.V. Morrison and G.F. Gilmore, eds.) Cambridge University Press, Cambridge, p. 297.

Arlot, J.-E., and Thuillot, W. (1988)."The coordination of the PHEMU85 international campaign," in Coordination of Observational projects in Astronomy (C. Jascheck and C. Sterken, eds.) Cambridge University Press, Cambridge, p. 171.

Arlot, J.-E., and Thuillot, W. (1993). "Eclipses and Mutual Events of the First Eight Saturnian Satellites during the 1993-1996 Period," Icarus 105, 427.

Arlot, J.-E., Thuillot, W., Sevre, F., Vu, D.T., and Descamps, P. (1990). "A new campaign of observation of the mutual events of the Galilean satellites in 1991," Astron. Astrophys. 236, L19.

Baum, W.A., Kreidl, T., Westphal, J.A., Danielson, G.E., Seidelmann, P.K., Pascu, D. and Currie, D.G. (1981). "Saturn's E Ring I. CCD Observations of March 1980," Icarus 47,84 . 
Beurle, K., Harper, D., Jones, D.H.P., Murray, C.D., Taylor, D.B. and Williams, I.P. (1993). "Preliminary analysis of CCD observations of Saturn's Satellites," Astron. Astrophys. 269, 564 .

Born, G.H., and Duxbury, T.C. (1975). "Phobos and Deimos ephemerides from Mariner 9 TV data," Cel. Mech. 12, 77.

Bosh, A.S., and Rivkin, A.S. (1995). "Saturn ring-plane crossing, May 1995," Bull. Amer. Astron. Soc. 27, 1131.

Brinkmann, R.T., and Millis, R.L. (1973). "Mutual Phenomena of Jupiter's Satellites in 1973-74," Sky and Telescope 45, 93.

Buratti, B.J., Dunbar, R.S., Tedesco, E.F., Gibson, J., Marcialis, R.L., Wong, F., Bennett, S., and Dobrovolskis, A. (1995). "Modeling Pluto-Charon Mutual Events II. CCD Observations with the 60-inch Telescope of Palomar Mountain," Astron. J. 110, 1405.

Campbell, D.B., Chandler, J.F., Ostro, S.J., Pettengill, G.H., and Shapiro, I.I. (1978). "Galilean satellites: 1976 radar results," Icarus 34, 254.

Christy, J. (1978). "1978 P 1," IAUC No.3241.

Colas, J.F. (1991)."L'observation des phenomenes mutuels avec une cible CCD," Note technique No.18, Bureau des Longitudes.

Colas, F. (1992). "Astrometric observations of Phobos and Deimos during the 1988 opposition of Mars," Astron. Astrophys. Supp. Ser. 96, 485.

Colas, F. (1994). "CCD observations at Pic du Midi Observatory: the techniques to be used," presented at PHESAT95 Workshop, 19-21 September 1994, Bucharest, Romania.

Colas, F. (1995). "Bureau des Longitudes and modern observations," presented at IAU Symposium 172, 3-8 July 1995, Paris, France.

Colas, F., and Arlot, J.-E. (1991). "Comparisons of observations of the Martian satellites made in 1988 with ephemerides," Astron. Astrophys. 252, 402.

Colas, J.F., and Buil, C. (1992). "First Earth-based observations of Neptune's satellite Proteus," Astron. Astrophys., 262, L13.

Currie, D., Dowling, D., Seidelmann, P.K., Pascu, D., Rohde, J.R., Wells, E., Storrs, A., and Zellner, B. (1994). "HST Images of the Planet Uranus: Satellites and Ring System," Bull. Amer. Astron. Soc., 26, 1376.

Dahn, C.C. (1994). personal comm.

Descamps, P. (1992). "Etude des effets de surface sur la réduction astrométrique des observations de phénomènes des satellites Galiléens de Jupiter," Thèse de Doctorat, Observatoire de Paris.

Descamps, P., Arlot, J.-E., Thuillot, W., Colas, J.F., Vu, D.T., Bouchet, P., and Hainaut, O. (1992). "Observations of the Volcanos of Io, Loki and Pele, made in 1991 at ESO During an Occultation by Europa," Icarus 100, 235.

Dourneau, G. (1982). "Observation of 2 mutual events involving the satellites of Saturn in April 1980," Astron. Astrophys. 112, 73.

Duxbury, T.C., and Callahan, J.D. (1988). "Phobos and Deimos astrometric observations from Viking," Astron. Astrophys. 201, 169.

Duxbury, T.C., and Callahan, J.D. (1989). "Phobos and Deimos astrometric observations from Mariner 9," Astron. Astrophys. 216, 284.

Goguen, J.D., Sinton, W.M., Matson, D.L., Howell, R.R., Dyck, H.M., Johnson, T.V., Brown, R.H., Veeder, G.J., Lane, A.L., Nelson, R.M., and McLaren, R.A. (1988). "Io Hot Spots: Infrared Photometry of Satellite Occultations," Icarus 76, 465.

Harper, D. (1994). "CCD observations at La Palma: results and projects," presented at PHESAT95 Workshop, 19-21 September 1994, Bucharest, Romania.

Harper, D. (1995). "CCD astrometry of Saturn's satellites in 1990-1994 from La Palma," presented at IAU Symposium 172, 3-8 July 1995, Paris, France.

Harris, F. (1995). personal comm. 
Jones, D.H.P. (1995). "Limitations on the accuracy possible in astrometric observations of satellites of the major planets," presented at IAU Symposium 172, 3-8 July 1995, Paris, France.

Le Campion, J.F., Montignac, G., Chauvet, F., Colin, J., Desbats, J.M., Dourneau, G., and Rapaport, M. (1992). "First CCD observations of mutual phenomena of the Galilean satellites," Astron. Astrophys. 266, 568.

Le Floch, J.C. (1994). "L'experience de l'Observatoire de Bordeaux dans les observations et le traitement des donnees sur les satellites de Saturne," presented at PHESAT95 Workshop, 19-21 September 1994, Bucharest, Romania.

Lieske, J. (1995a). "Making sense out of the 1985 and 1991 mutual events," Bull. Amer. Astron. Soc. 27, 1197.

Lieske, J.H. (1995b). "Galilean satellites," presented at IAU Symposium 172, 3-8 July 1995, Paris, France.

Lindegren, L. (1980). "Atmospheric Limitations of Narrow-field Optical Astrometry," Astron. Astrophys. 89, 41.

Lopez-Garcia, A. (1994). "CCD observations of major planet satellites," presented at PHESAT95 Workshop, 19-21 September 1994, Bucharest, Romania.

Mallama, A. (1991). "Light Curve Model for the Galilean Satellites during Jovian Eclipse," Icarus 92, 324.

Mallama, A. (1992a). "Astrometry of the Galilean Satellites from Mutual Eclipses and Occultations," Icarus 95, 309.

Mallama, A. (1992b). "CCD Photometry for Jovian Eclipses of the Galilean Satellites," Icarus 97, 298.

Mallama, A. (1995). "Detection of Very High Altitude Fall-out from the Comet Shoemaker-Levy 9 Explosions in Jupiter's Atmosphere," J. Geophys. Res.- Planets (submitted).

Millis, R.L. (1974). "Mutual Occultations and Eclipses of the Galilean Satellites," Bull. Amer. Astron. Soc. 6, 382.

Monet, A.K.B. (1993). "Ground-based Astrometry for the Galileo Mission," Bull. Amer. Astron. Soc. 25, 1234.

Monet, D.G., and Monet, A.K.B. (1992). "Galilean Satellite Astrometry," inter-office memo, 22 Oct. 1992. U.S. Naval Observatory, Flagstaff Station.

Morando, B., and Descamps, P. (1994). "Some results obtained during the 1991 campaign of the observation of the mutual events of the Galilean satellites," in Galactic and Solar System Optical Astrometry (L.V. Morrison and G.F. Gilmore, eds.) Cambridge University Press, Cambridge, p. 329.

Muhleman, D.O., Berge, G.L., Rudy, D., and Niell, A.E. (1986). "Precise VLA Positions and Flux-Density Measurements of the Jupiter System," Astron. J. 92, 1428.

Nicholson, P.D., Hamilton, D.P., Matthews, K., and Yoder, C.F. (1992). "New Observations of Saturn's Coorbital Satellites," Icarus 100, 464.

Nicholson, P.D., and Matthews, K. (1991). "Near-Infrared Observations of the Jovian Ring and Small Satellites," Icarus 93, 331.

Null, G.W., Owen, W.M., and Synnott, S.P. (1993). "Masses and densities of Pluto and Charon," Astron. J. 105, 2319.

Owen, W.M. (1995). personal comm.

Pascu, D. (1977). "Astrometric Techniques for the Observation of Planetary Satellites," in Planetary Satellites, (J.A. Burns, ed.) University of Arizona Press, Tucson, p. 63.

Pascu, D. (1979). "The Naval Observatory Program for the Astrometric Observation of Planetary Satellites," in Natural and Artificial Satellite Motion, (P.E. Nacozy and S. Ferraz-Mello, eds.) University of Texas Press, Austin, p. 17.

Pascu, D. (1994a). "An appraisal of the USNO program for photographic astrometry of bright planetary satellites," in Galactic and Solar System Optical Astrometry, ( L.V. Morrison and G.F. Gilmore, eds.) Cambridge University Press, Cambridge, p. 304. 
Pascu, D. (1994b). "CCD observations of planetary satellites at the U.S. Naval Observatory," presented at PHESAT95 Workshop, 19-21 September 1994, Bucharest, Romania.

Pascu, D., Adler, C.A. and Bloomfield, J.F. (1991). "An Analysis of Photographic Astrometric Observations of the Galilean Moons," Bull. Amer. Astron. Soc. 23, 1255

Pascu, D., Colas, J.F., and Rohde, J.R. (1992b). "Astrometric CCD Observations of Thebe (JXIV)," Bull. Amer. Astron. Soc. 24, 1059.

Pascu, D., Panossian, S.P., Schmidt, R.E., Seidelmann, P.K., and Hershey, J.L. (1992a). "B, V Photometry of Thebe (JXIV)," Icarus 98, 38.

Pascu, D., and Rohde, J.R. (1993). "BVRI Photometry of Helene (SXII), Telesto (SXIII), and Calypso (SXIV)," Bull. Amer. Astron. Soc. 25, 1302.

Pascu, D., Rohde, J.R., and Colas, J.F. (1993). "Relative BVRI Photometry of Thebe (JXIV) and Amalthea (JV)," Bull. Amer. Astron. Soc. 25, 1114.

Pascu, D., Rohde, J.R., and Foechterle (1994a). "B, V Photometry of Helene (SXII)," Bull. Amer. Astron. Soc. 26, 1163.

Pascu, D., Rohde, J.R., Seidelmann, P.K., Currie, D.G., Dowling, D.M., Wells, E., Kowal, C., Zellner, B., and Storrs, A. (1995). "HST Astrometry of the Uranian Inner Satellite System,” Bull. Amer. Astron. Soc. 27, 829.

Pascu, D., and Schmidt, R.E. (1990). "Photographic Positional Observations of Saturn," Astron. J. 99, 1974.

Pascu, D., Seidelmann, P.K., Baum, W.A. and Schmidt, R.E. (1983). "Observations of Faint Planetary Satellites with a Charge- Coupled Device," in The Motion of Planets and Natural and Artificial Satellites, (S. Ferraz-Mello and P.E. Nacozy, eds.) Universidade de São Paulo, Brasil, p. 253.

Pascu, D., Seidelmann, P.K., Schmidt, R.E., Santoro, E.J. and Hershey, J.L. (1987). "Astrometric CCD Observations of Miranda: 1981-1985," Astron. J. 93, 963.

Rapaport, M. (1994). "Observations of Saturn's satellites made with the CCD camera of Bordeaux Observatory; comparison with the theories," presented at the PHESAT95 Workshop, 19-21 September 1994, Bucharest, Romania.

Rohde, J.R., and Pascu, D. (1993). "Astrometric Observations of Helene (SXII), Telesto (SXIII), and Calypso (SXIV)," Bull. Amer. Astron. Soc. 25, 1235.

Rohde, J.R., and Pascu, D. (1994). "CCD Astrometry of Helene (SXII), Telesto (SXIII), and Calypso (SXIV): 1993 Observations," Bull. Amer. Astron. Soc. 26, 1024.

Seidelmann, P.K. (1977). "Tabulations of Satellite Positional Observations and their Discussion," in Planetary Satellites (J.A. Burns, ed.), University of Arizona Press, Tucson, p. 533.

Seidelmann, P.K. (1979). "Planetary Satellites, a Review of the Past and Assessment of the Future," in Natural and Artificial Satellite Motion (P.E. Nacozy and S. FerrazMello, eds.), University of Texas Press, Austin, p. 3.

Showalter, M.R., Nicholson, P.D., Danielson, G.E., Dones, L., French, R.G., Larson, S., Lissauer, J., McGhee, C., Seitzer, P., and Sicardy, B. (1995). "HST Observations of Saturn during the August 1995 Ring Plane Crossing," Bull. Amer. Astron. Soc. 27, 1131.

Tholen, D.J. and Buie, M.W. (1995). "The orbit of Charon from Hubble Space Telescope observations," presented at IAU Symposium 172, 3-8 July 1995, Paris, France.

Veiga, C.H. and Vieira Martins, R. (1994). "A method to define a reference system for the reduction of astrometric positions of natural satellites," presented at PHESAT95 Workshop, 19-21 September 1994, Bucharest, Romania.

Veillet, C., and Ratier, G. (1980). "Astrometric Study of the Uranus Satellite Miranda," Astron. Astrophys. 89, 342.

Viateau, B., and Rapaport, M. (1995). "Observations astrometriques a l'Observatoire de Bordeaux," presented at IAU Symposium 172, 3-8 July 1995, Paris, France.

Vieira Martins, R., and Veiga, C.H. (1995). "Astrometric observations of faint satellites," presented at IAU Symposium 172, 3-8 July 1995, Paris, France. 
Zamarashkin, K.N., Kirsanov, N.O., Kisseleva, T.P., Kisselev, A.A. and Batrakov, J.V. (1994). "CCD astrometric observations of the Saturnian satellites system on the 26inch refractor at Pulkovo to be made in 1995-1996 campaign and their comparison with parallel photographic observations," presented at PHESAT95 Workshop, 19-21 September 1994, Bucharest, Romania.

Zellner, B, Seidelmann, P.K., Pascu, D., Kowal, C., Wells, E., and Currie, D.G. (1994). "Recovery of Inner Satellites of Uranus," Bull. Amer. Astron. Soc. 26, 1163. 